\title{
Dominance of Prevotella and low abundance of classical ruminal bacterial species in the bovine rumen revealed by relative quantification real-time PCR
}

\author{
David M. Stevenson • Paul J. Weimer
}

Published online: 29 May 2009

(C) Springer-Verlag 2009

Erratum to: Appl Microbiol Biotechnol DOI 10.1007/s00253-006-0802-y

This article unfortunately contained a mistake. The strain designations of two species, Prevotella brevis and Prevotella bryantii, were inadvertently reversed in Table 2 . The names and sequences of the corresponding species-specific primers were not in error. The corrected table appears below.

The online version of the original article can be found at http://dx.doi. org/10.1007/s00253-006-0802-y.

D. M. Stevenson $\cdot$ P. J. Weimer

Agricultural Research Service, U.S. Department of Agriculture, United States Dairy Forage Research Center,

Madison, WI 53706, USA

P. J. Weimer $(\square)$

Department of Bacteriology, University of Wisconsin-Madison,

Madison, WI 53706, USA

e-mail: pjweimer@wisc.edu 
Table 2 PCR primers used in this study

\begin{tabular}{|c|c|c|c|c|c|}
\hline Target taxon and specific strain tested & Primer set & Primer sequences & $\mathrm{Tm}^{\mathrm{a}}$ & $E^{b}$ & Amplicon $\mathrm{Tm}^{\mathrm{c}}$ \\
\hline \multirow[t]{2}{*}{ Butyrivibrio fibrisolvens $\mathrm{H} 17 \mathrm{c}$} & ButFib2F & ACCGCATAAGCGCACGGA & 62 & 1.98 & 74 \\
\hline & ButFib2R & CGGGTCCATCTTGTACCGATAAAT & 61 & & \\
\hline \multirow[t]{2}{*}{ Eubacterium ruminantium GA195 } & EubRum2F & CTCCCGAGACTGAGGAAGCTTG & 61 & 1.97 & 80 \\
\hline & EubRum2R & GTCCATCTCACACCACCGGA & 60 & & \\
\hline \multirow[t]{2}{*}{ Fibrobacter succinogenes $\mathrm{S} 85$} & FibSuc3F & GCGGGTAGCAAACAGGATTAGA & 59 & 1.93 & 77 \\
\hline & FibSuc3R & CCCCCGGACACCCAGTAT & 59 & & \\
\hline \multirow[t]{2}{*}{ Megasphaera elsdenii $\mathrm{T} 81$} & MegEls2F & AGATGGGGACAACAGCTGGA & 59 & 1.97 & 79 \\
\hline & MegEls2R & CGAAAGCTCCGAAGAGCCT & 58 & & \\
\hline \multirow[t]{2}{*}{ Prevotella brevis GA33 } & PreBre1F & GGTTTCCTTGAGTGTATTCGACGTC & 61 & 1.98 & 82 \\
\hline & PreBre1R & CTTTCGCTTGGCCGCTG & 60 & & \\
\hline \multirow[t]{2}{*}{ Prevotella bryantii $\mathrm{B}_{1} 4$} & PreBry2F & AGCGCAGGCCGTTTGG & 61 & 1.95 & 83 \\
\hline & PreBry2R & GCTTCCTGTGCACTCAAGTCTGAC & 61 & & \\
\hline \multirow[t]{2}{*}{ Prevotella ruminicola 23} & PreRum1F & GAAAGTCGGATTAATGCTCTATGTTG & 58 & 1.93 & 71 \\
\hline & PreRum1R & CATCCTATAGCGGTAAACCTTTGG & 59 & & \\
\hline \multirow[t]{2}{*}{ Ruminobacter amylophilus $\mathrm{H} 18$} & RumAmy2F & CTGGGGAGCTGCCTGAATG & 60 & 1.96 & 82 \\
\hline & RumAmy2R & GCATCTGAATGCGACTGGTTG & 60 & & \\
\hline \multirow[t]{2}{*}{ Ruminococcus albus 7} & RumAlb3F & TGTTAACAGAGGGAAGCAAAGCA & 60 & 1.85 & 75 \\
\hline & RumAlb3R & TGCAGCCTACAATCCGAACTAA & 59 & & \\
\hline \multirow[t]{2}{*}{ Ruminococcus flavefaciens FD-1 } & RumFla3F & TGGCGGACGGGTGAGTAA & 60 & 1.79 & 78 \\
\hline & RumFla3R & TTACCATCCGTTTCCAGAAGCT & 60 & & \\
\hline \multirow[t]{2}{*}{ Selenomonas ruminantium D } & SelRum2F & CAATAAGCATTCCGCCTGGG & 61 & 1.95 & 82 \\
\hline & SelRum2R & TTCACTCAATGTCAAGCCCTGG & 61 & & \\
\hline \multirow[t]{2}{*}{ Streptococcus bovis JB1 } & StrBov2F & TTCCTAGAGATAGGAAGTTTCTTCGG & 59 & 1.95 & 82 \\
\hline & StrBov2R & ATGATGGCAACTAACAATAGGGGT & 59 & & \\
\hline \multirow[t]{2}{*}{ Succinivibrio dextrinosolvens $22 \mathrm{~b}$} & SucDex1F & CGTCAGCTCGTGTCGTGAGA & 60 & 1.95 & 80 \\
\hline & SucDex1R & CCCGCTGGCAACAAAGG & 60 & & \\
\hline \multirow[t]{2}{*}{ Domain Bacteria $^{\mathrm{d}}$} & BAC $338 \mathrm{~F}$ & ACTCCTACGGGAGGCAG & 52 & $1.92-$ & $\mathrm{f}$ \\
\hline & BAC $805 \mathrm{R}$ & GACTACCAGGGTATCTAATCC & 48 & $2.01^{\mathrm{e}}$ & \\
\hline \multirow[t]{2}{*}{ Genus Prevotella ${ }^{\mathrm{g}}$} & PreGen4F & GGTTCTGAGAGGAAGGTCCCC & 60 & 1.92 & 83 \\
\hline & PreGen4R & TCCTGCACGCTACTTGGCTG & 61 & & \\
\hline
\end{tabular}

All primers were designed for this study except for BAC338F and BAC805R, which were designed by Yu et al. (2005).

a Tm values calculated using Primer Express software (Applied Biosystems, Foster City, CA). Tm varied among species

${ }^{\mathrm{b}} E$ represents the efficiency calculated from an amplification of a dilution series of target DNA as described in the text. For the domain-level eubacterial primer, the indicated range spans the efficiencies determined against all of the indicated genus (Prevotella) or species level taxa listed. An efficiency of 2.0 is equivalent to $100 \%$ of theoretical amplification per PCR cycle.

${ }^{c}$ Amplicon Tm calculated from the dissociation protocol run on the resultant amplicon generated with the selected primer against the pure species indicated.

d Domain-level primers.

e Efficiency values varied among species within this range.

${ }^{\mathrm{f}} \mathrm{Tm}$ values varied among species. Five strains were tested, which included Butyrivibrio fibrisolvens $\left(\mathrm{Tm}=82^{\circ} \mathrm{C}\right)$, Eubacterium ruminantium $\left(\mathrm{Tm}=83^{\circ} \mathrm{C}\right)$, Fibrobacter succinogenes $\left(\mathrm{Tm}=85^{\circ} \mathrm{C}\right)$, Ruminococcus flavefaciens $\left(\mathrm{Tm}=83^{\circ} \mathrm{C}\right)$, and Streptococcus bovis $\left(\mathrm{Tm}=82^{\circ} \mathrm{C}\right)$.

g Genus-level primers. P. brevis GA33 was used as test strain. 